\title{
Extending Automated Compositional Verification to the Full Class of Omega-Regular Languages ${ }^{\star}$
}

\author{
Azadeh Farzan ${ }^{1}$, Yu-Fang Chen ${ }^{2}$, Edmund M. Clarke ${ }^{1}$, Yih-Kuen Tsay ${ }^{2}$, \\ and Bow-Yaw Wang ${ }^{3}$ \\ ${ }^{1}$ Carnegie Mellon University \\ ${ }^{2}$ National Taiwan University \\ ${ }^{3}$ Academia Sinica
}

\begin{abstract}
Recent studies have suggested the applicability of learning to automated compositional verification. However, current learning algorithms fall short when it comes to learning liveness properties. We extend the automaton synthesis paradigm for the infinitary languages by presenting an algorithm to learn an arbitrary regular set of infinite sequences (an $\omega$-regular language) over an alphabet $\Sigma$. Our main result is an algorithm to learn a nondeterministic Büchi automaton that recognizes an unknown $\omega$-regular language. This is done by learning a unique projection of it on $\Sigma^{*}$ using the framework suggested by Angluin for learning regular subsets of $\Sigma^{*}$.
\end{abstract}

\section{Introduction}

Compositional verification is an essential technique for addressing the state explosion problem in Model Checking [17811. Most compositional techniques advocate proving properties of a system by checking properties of its components in an assume-guarantee style. The essential idea is to model check each component independently by making an assumption about its environment, and then discharge the assumption on the collection of the rest of the components. In the paradigm of automated compositional reasoning through learning [8], system behaviors and their requirements are formalized as regular languages. Assumptions in premises of compositional proof rules are often regular languages; their corresponding finite-state automata can therefore be generated by learning techniques for regular languages.

In automated compositional reasoning, a compositional proof rule is chosen $a$ priori. The rule indicates how a system can be decomposed. Below is an example of a simple rule:

$$
\frac{M_{2} \models A \quad M_{1} \| A \models P}{M_{1} \| M_{2} \models P}
$$

\footnotetext{
* This research was sponsored by the iCAST project of the National Science Council, Taiwan, under the grant no. NSC96-3114-P-001-002-Y and the Semiconductor Research Corporation (SRC) under the grant no. 2006-TJ-1366.
} 
for two components $M_{1}$ and $M_{2}$, and assumption $A$, and a property $P$. Intuitively, this rule says that if $M_{2}$ guarantees $A$, and $M_{1}$ guarantees $P$ in an environment that respects $A$, then the system composed of $M_{1}$ and $M_{2}$ guarantees $P$. The goal is to automatically generate the assumption $A$ by learning. One naturally wishes to verify all sorts of properties using this framework. However, all existing algorithms fall short when it comes to learning assumptions which involve liveness properties. In this paper, we present an algorithm that fills this gap and extends the learning paradigm to the full class of $\omega$-regular languages. Soundness and completeness of the above proof rule with respect to liveness properties remains intact since $\omega$-regular languages share the required closure properties of regular languages. Automation can be achieved following the framework of 8]. See 9] for a more detailed discussion.

The active learning model used in automated compositional reasoning involves a teacher who is aware of an unknown language, and a learner whose goal is to learn that language. The learner can put two types of queries to the teacher. A membership query asks if a string belongs to the unknown language. An equivalence query checks whether a conjecture automaton recognizes the unknown language. The teacher provides a counterexample if the conjecture is incorrect [2]. More specifically, in the process of learning an assumption, an initial assumption is generated by the learner through a series of membership queries. An equivalence query is then made to check if the assumption satisfies premises of the compositional proof rule. If it does, the verification process terminates with success. Otherwise, the learner refines the assumption by the returned counterexample and more membership queries. Since the weakest assumption either establishes or falsifies system requirements, the verification process eventually terminates when the weakest assumption is attained. A novel idea in [8] uses model checkers to resolve both membership and equivalence queries automatically. By using Angluin's L* 2] algorithm, the verification process can be performed without human intervention.

The product of the learning algorithm $\mathrm{L}^{*}$ is a deterministic finite-state automaton recognizing the unknown regular language [2]. By the Myhill-Nerode Theorem, the minimal deterministic finite-state automaton can be generated from the equivalence classes defined by the coarsest right congruence relation of any regular language [13. The $L^{*}$ algorithm computes the equivalence classes by membership queries, and refines them with counterexamples returned by equivalence queries. It can, in fact, infer the minimal deterministic finite-state automaton for any unknown regular language with a polynomial number of queries in the size of the target automaton. The upper bound was later improved in [18].

Unfortunately, the $\mathrm{L}^{*}$ algorithm cannot be directly generalized to learn $\omega$ regular languages. Firstly, deterministic Büchi automata are less expressive than general Büchi automata. Inferred deterministic finite-state automata require more than the Büchi acceptance condition to recognize arbitrary $\omega$-regular languages. Secondly, equivalence classes defined by the coarsest right congruence relation over an $\omega$-regular language do not necessarily correspond to the states of its automaton. The $\omega$-regular language $(a+b)^{*} a^{\omega}$ has only one equivalence 
class. Yet, there is no one-state $\omega$-automaton with Büchi, Rabin, Streett, or even Muller acceptance conditions that can recognize this language.

Maler and Pnueli 14 made an attempt to generalize $\mathrm{L}^{*}$ for the $\omega$-regular languages. Their algorithm, $\mathrm{L}^{\omega}$, learns a proper subclass of $\omega$-regular languages which is not expressive enough to cover liveness properties. This restricted class has the useful property of being uniquely identifiable by the syntactic right congruence. Thus, $\mathrm{L}^{\omega}$ has the advantage of generating the minimal deterministic Muller automaton (isomorphic to the syntactic right congruence) recognizing a language in the restricted class. The syntactic right congruence, however, cannot be used to identify an arbitrary $\omega$-regular language. Attempts to use more expressive congruences 321 have been unsuccessful.

Our main ideas are inspired by the work of Calbrix, Nivat, and Podelski [5]. Consider ultimately periodic $\omega$-strings of the form $u v^{\omega}$. Büchi [4] observed that the set of ultimately periodic $\omega$-strings characterizes $\omega$-regular languages; two $\omega$-regular languages are in fact identical if and only if they have the same set of ultimately periodic $\omega$-strings. Calbrix et al. 5] show that the finitary language $\left\{u \$ v \mid u v^{\omega} \in L\right\}$ (where $\$$ is a fresh symbol) is regular for any $\omega$-regular language $L$. These properties help uniquely identify a Büchi automaton for the regular language corresponding to ultimately periodic $\omega$-strings of an arbitrary $\omega$-regular language. We develop a learning algorithm for the regular language $\left\{u \$ v \mid u v^{\omega} \in L\right\}$ through membership and equivalence queries on the unknown $\omega$-regular language $L$. A Büchi automaton accepting $L$ can hence be constructed from the finite-state automaton generated by our learning algorithm.

\section{Preliminaries}

Let $\Sigma$ be a finite set called the alphabet. A finite word over $\Sigma$ is a finite sequence of elements of $\Sigma$. An empty word is represented by $\epsilon$. For two words $u=u_{1} \ldots u_{n}$ and $v=v_{1} \ldots v_{n}$, define $u v=u_{1} \ldots u_{n} v_{1} \ldots v_{m}$. For a word $u, u^{n}$ is recursively defined as $u u^{n-1}$ with $u^{0}=\epsilon$. Define $u^{+}=\bigcup_{i=1}^{\infty}\left\{u^{i}\right\}$, and $u^{*}=\{\epsilon\} \cup u^{+}$. An infinite word over $\Sigma$ is an infinite sequence of elements of $\Sigma$. For a finite word $u$, define the infinite word $u^{\omega}=u u \ldots u \ldots$ Operators,$+ *$, and $\omega$ are naturally extended to sets of finite words.

A word $u$ is a prefix (resp. suffix) of another word $v$ if and only if there exists a word $w \in \Sigma^{*}$ such that $v=u w$ (resp. $v=w u$ ). A set of words $S$ is called prefix-closed (resp. suffix-closed) if and only if for all $v \in S$, if $u$ is a prefix (resp. suffix) of $v$ then $u \in S$.

The set of all finite words on $\Sigma$ is denoted by $\Sigma^{*} . \Sigma^{+}$is the set of all nonempty words on $\Sigma$; therefore, $\Sigma^{+}=\Sigma^{*} \backslash\{\epsilon\}$. Let $u$ be a finite word. $|u|$ is the length of word $u$ with $|\epsilon|=0$. The set of all infinite words on $\Sigma$ is denoted by $\Sigma^{\omega}$. A language is a subset of $\Sigma^{*}$, and an $\omega$-language is a subset of $\Sigma^{\omega}$.

A finite automaton $\mathcal{A}$ is a tuple $(\Sigma, Q, I, F, \delta)$ where $\Sigma$ is an alphabet, $Q$ is a finite set of states, $I \subseteq Q$ is a set of initial states, $F \subseteq Q$ is a set of final states, and $\delta \subseteq Q \times \Sigma \times Q$ is the transition relation. A finite word $u=u_{1} \ldots u_{n}$ is accepted by $\mathcal{A}$ if and only if there exists a sequence $q_{i_{0}} u_{1} q_{i_{1}} u_{2} \ldots u_{n} q_{i_{n}}$ such that 
$q_{i_{0}} \in I, q_{i_{n}} \in F$, and for all $j$, we have $q_{i_{j}} \in Q$ and $\left(q_{i_{j-1}}, u_{j}, q_{i_{j}}\right) \in \delta$. Define $L(\mathcal{A})=\{u \mid u$ is accepted by $\mathcal{A}\}$. A language $L \subseteq \Sigma^{*}$ is regular if and only if there exists an automaton $\mathcal{A}$ such that $L=L(\mathcal{A})$.

A Büchi automaton has the same structure as a finite automaton, except that it is intended for recognizing infinite words. An infinite word $u=u_{1} \ldots u_{n} \ldots$ is accepted by a Büchi automaton $\mathcal{A}$ if and only if there exists a sequence $q_{i_{0}} u_{1} q_{i_{1}} u_{2} \ldots u_{n} q_{i_{n}} \ldots$ such that $q_{i_{0}} \in I, q_{i_{j}} \in Q$ and $\left(q_{i_{j-1}}, u_{j}, q_{i_{j}}\right) \in \delta$ (for all $j$ ), and there exists a state $q \in F$ such that $q=q_{i_{j}}$ for infinitely many $j$ 's. Again, define $L(\mathcal{A})=\{u \mid u$ is accepted by $\mathcal{A}\}$. An $\omega$-language $L \subseteq \Sigma^{\omega}$ is $\omega$ regular if and only if there exists a Büchi automaton $\mathcal{A}$ such that $L=L(\mathcal{A})$. For an $\omega$-language $L$, let $U P(L)=\left\{u v^{\omega} \mid u \in \Sigma^{*}, v \in \Sigma^{+}, u v^{\omega} \in L\right\}$. Words of the form $u v^{\omega}$ are called the ultimately periodic. Let $\alpha$ be an ultimately periodic word. A word $v \in \Sigma^{+}$is a period of $\alpha$ if there exists a word $u \in \Sigma^{*}$ such that $\alpha=u v^{\omega}$.

Theorem 1. (Büchi) [4] Let $L$ and $L^{\prime}$ be two $\omega$-regular languages. $L=L^{\prime}$ if and only if $U P(L)=U P\left(L^{\prime}\right)$.

The above theorem implies that the set of ultimately periodic words of an $\omega$ regular language $L$ uniquely characterizes $L$. Define $L_{\$}$ (read regular image of $L)$ on $\Sigma \cup\{\$\}$ as

$$
L_{\$}=\left\{u \$ v \mid u v^{\omega} \in L\right\} .
$$

Intuitively, the symbol $\$$ marks the beginning of the period and separates it from the prefix of the $\omega$-word $u v^{\omega}$. Note that $L_{\$} \subseteq \Sigma^{*} \$ \Sigma^{+}$. We can then say that $L_{\$}$ uniquely characterizes $L$.

Theorem 2. (Büchi) [4] If $L$ is an $\omega$-regular language, then there exist regular languages $L_{1}, \ldots, L_{n}$ and $L_{1}^{\prime}, \ldots, L_{n}^{\prime}$ such that $L=\bigcup_{i=1}^{n} L_{i}\left(L_{i}^{\prime}\right)^{\omega}$.

Theorem 3. (Calbrix, Nivat, and Podelski)[5] $L_{\$}$ is regular.

Moreover, one can show that the syntactic congruence of the regular language $L_{\S}$ and Arnold's congruence [3] for $L$ coincide on the set $\Sigma^{+}[6]$.

\section{Ultimately Periodic Words}

Define an equivalence relation on the words in $\Sigma^{*} \$ \Sigma^{+}$:

Definition 1. The equivalence relation $\stackrel{\circ}{=}$ on $\Sigma^{*} \$ \Sigma^{+}$is defined by:

$$
u \$ v \stackrel{\circ}{\circ} \$ v^{\prime} \Longleftrightarrow u v^{\omega}=u^{\prime} v^{\omega}
$$

$u, u^{\prime} \in \Sigma^{*}$ and $v, v^{\prime} \in \Sigma^{+}$.

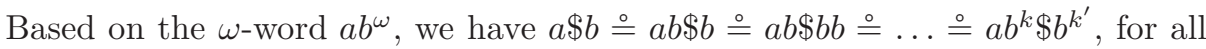
$k, k^{\prime}$. Therefore, the equivalence class $[a \$ b] \triangleq$ is equal to the set of words $a b^{*} \$ b^{+}$. 
Definition 2. An equivalence relation $\equiv$ saturates a language $L$ if and only if for two words $u$ and $v$, where $u \equiv v$, we have $u \in L$ implies $v \in L$.

Let $L$ be an $\omega$-regular language, and $L_{\$}$ its corresponding regular language as defined above. Let $u \$ v$ be a word in $L_{\$}$ and $u^{\prime} \$ v^{\prime} \in \Sigma^{*} \$ \Sigma^{+}$such that $u \$ v \stackrel{\circ}{=}$ $u^{\prime} \$ v^{\prime}$. Since $u v^{\omega}=u^{\prime} v^{\prime \omega}$, we have $u^{\prime} v^{\prime \omega} \in L$, and therefore (by definition) $u^{\prime} \$ v^{\prime} \in$ $L_{\$}$. This implies the following Proposition:

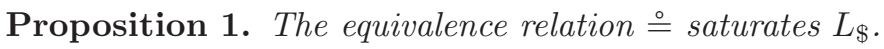

Let $R \subseteq \Sigma^{*} \$ \Sigma^{+}$be a regular language. Proposition 1 suggests that saturating $\stackrel{\circ}{=}$ is a necessary condition for $R$ to be $L_{\$}$ for some $\omega$-regular language $L$. The interesting point is that one can show that it is sufficient as well. This can be done by constructing a Büchi automaton $\mathcal{B}$ that recognizes $L$ directly from the automaton $\mathcal{A}$ recognizing $R$ [5]. Since this construction is used in our algorithm, we describe it here. We first need the following lemma:

Lemma 1. (Calbrix, Nivat, and Podelski) [5] Let $L, L^{\prime} \subseteq \Sigma^{*}$ be two regular languages such that $L L^{\prime *}=L$ and $L^{\prime+}=L^{\prime}$. Then, $\alpha \in U P\left(L L^{\prime \omega}\right)$ if and only if there exist $u \in L$ and $v \in L^{\prime}$ such that $\alpha=u v^{\omega}$.

Let $R \subseteq \Sigma^{*} \$ \Sigma^{+}$be a regular language. Let $\mathcal{A}=(\Sigma \cup\{\$\}, Q, I, F, \delta)$ be a deterministic automaton recognizing $R$. Define $Q_{\$}$ to be the set of states that can be reached by starting in an initial state and reading the part of a word $u \$ v \in M$ that precedes the $\$$. Formally,

$$
Q_{\$}=\left\{q \in Q \mid \exists u \$ v \in R, \exists q_{i} \in I, q=\delta\left(q_{i}, u\right)\right\}
$$

For each state $q \in Q_{\$}$, let

$$
\begin{aligned}
& M_{q}=\left\{u \mid \exists q_{i} \in I, \delta\left(q_{i}, u\right)=q\right\} \\
& N_{q}=\left\{v \mid \exists q_{f} \in F, \delta(q, \$ v)=q_{f}\right\} .
\end{aligned}
$$

For each $q, M_{q}$ and $N_{q}$ are regular languages; one can easily construct an automaton accepting each by modifying $\mathcal{A}$. Moreover, the definitions of $M_{q}$ and $N_{q}$ along side the fact $R \subseteq \Sigma^{*} \$ \Sigma^{+}$, implies that $R=\bigcup_{q \in Q_{\$}} M_{q} \$ N_{q}$.

Next, we partition $N_{q}$ based on the final states of the automaton. For each final state $q_{f} \in F$ and $q \in Q_{\$}$, let the regular language $N_{q, q_{f}}$ be

$$
N_{q, q_{f}}=\left\{v \mid \delta(q, v)=q \wedge \delta(q, \$ v)=q_{f} \wedge \delta\left(q_{f}, v\right)=q_{f}\right\}
$$

Finally, for the regular language $R \subseteq \Sigma^{*} \$ \Sigma^{+}$, we define the $\omega$-regular language $\omega(R)$ as

$$
\omega(R)=\bigcup_{\left(q, q_{f}\right) \in Q_{\$} \times F} M_{q} N_{q, q_{f}}^{\omega} .
$$

We call this language $\omega(R)$ to indicate the fact that it is the corresponding $\omega$-regular language of $R$. Next we show that $\omega(R)$ is the $\omega$-regular language whose regular imgage is indeed $R$. The following theorem states this result: 
Theorem 4. Let $R \subseteq \Sigma^{*} \$ \Sigma^{+}$be a regular language that is saturated by Then, there exists an $\omega$-regular language $L$ such that $R=L_{\$}$.

Proof. See 9 for the proof.

In fact, $L=\omega(R)$ in the above theorem. One can directly build a Büchi automaton recognizing $L$ from $\mathcal{A}$. The set $Q_{\$}$ can be effectively computed. For each state $q \in Q_{\$}$, the language $M_{q}$ is recognized by the automaton $(\Sigma, Q, I,\{q\}, \delta)$. For each final state $q_{f}$, the language $N_{q, q_{f}}$ is the intersection of the languages $L(\Sigma, Q,\{q\},\{q\}, \delta), L\left(\Sigma, Q,\{\delta(q, \$)\},\left\{q_{f}\right\}, \delta\right)$, and $L\left(\Sigma, Q,\left\{q_{f}\right\},\left\{q_{f}\right\}, \delta\right)$. For each pair $\left(q, q_{f}\right)$, once we have DFAs recognizing $M_{q}$ and $N_{q, q_{f}}$, we can easily construct 11 a Büchi automaton recognizing $M_{q} N_{q, q_{f}}^{\omega}$. The Büchi automaton recognizing $L$ is the union of these automata. Each $M_{q}^{q} N_{q, q_{f}}^{\omega}$ is recognized by an automaton of size at most $|\mathcal{A}|+|\mathcal{A}|^{3}$, which means that $L$ is recognized by an automata of size at most $|\mathcal{A}|^{3}+|\mathcal{A}|^{5}$.

A question that naturally arises is what can one say about the result of the above construction if $R$ is not saturated by $\stackrel{\circ}{=}$. As we will see in Section 4 we need to construct Büchi automata from DFAs guessed in the process of learning

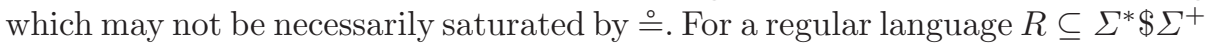
which is not saturated by $\stackrel{\circ}{=}$ and $L=\bigcup_{\left(q, q_{f}\right) \in Q_{\$} \times F} M_{q} N_{q, q_{f}}^{\omega}$, it is not necessarily the case that $R=L_{\$}$ (compare with the statement of Theorem 4 ). For example, $R=\{a \$ b\}$ is not saturated by $\stackrel{\circ}{=}$ since it contains an element of the class $[a \$ b] \triangleq$ (namely, $a \$ b$ ), but does not contain the whole class (which is the set $a b^{*} \$ b^{+}$). But, $L$ has a number of essential properties:

Proposition 2. Let $R=U_{\$}$ for some arbitrary $\omega$-regular language $U$. Then, we have $\omega(R)=U$ (defined by (4)).

Proof. Direct consequence of Theorem 4 .

Proposition 3. Assume $R \subseteq \Sigma^{*} \$ \Sigma^{+}$is a regular language. Let $[u \$ v] \triangleq$ denote the equivalence class of the word $u \$ v$ by the relation $\stackrel{\circ}{=}$ For each pair of words $(u, v) \in \Sigma^{*} \times \Sigma^{+}$, if $[u \$ v] \triangleq \cap R=\emptyset$ then $u v^{\omega} \notin \omega(R)$.

Proof. If $u v^{\omega} \in \omega(R)$, there exist a string $u^{\prime}$ in some $M_{q}$ and a string $v^{\prime}$ in some $N_{q, q_{f}}$ such that $u^{\prime} v^{\prime \omega}=u v^{\omega}$ (Lemma 1). Since $u^{\prime}$ is in $M_{q}$ and $v^{\prime}$ is in $N_{q, q_{f}}$, we have $u^{\prime} \$ v^{\prime}$ in $R$. Because $u^{\prime} v^{\prime \omega}=u v^{\omega}$, we have $u^{\prime} \$ v^{\prime} \in[u \$ v]_{\circ}$, which contradicts $[u \$ v] \triangleq \cap=\emptyset$.

Proposition 4. Assume $R \subseteq \Sigma^{*} \$ \Sigma^{+}$is a regular language. For each pair of words $(u, v) \in \Sigma^{*} \times \Sigma^{+}$, if $[u \$ v]_{\circ} \subseteq R$ then $u v^{\omega} \in \omega(R)$.

Proof. If $[u \$ v]_{\triangleq} \subseteq R$, we can find $k$ and $k^{\prime}$ satisfying $u v^{k}\left(v^{k^{\prime}}\right)^{\omega} \in \omega(R)$ (follows from proof of Theorem 4). Since $u v^{\omega}=u v^{k}\left(v^{k^{\prime}}\right)^{\omega}$, we have $u v^{\omega} \in \omega(R)$.

${ }^{1}$ One can connect the final states of $\mathcal{A}\left(M_{q}\right)$ to the initial states of $\mathcal{A}^{\omega}\left(N_{q, q_{F}}\right)$ by $\epsilon$ transitions, and let the final states of $N_{q, q_{f}}$ be the final states of the resulting Büchi automaton. $\mathcal{A}^{\omega}\left(N_{q, q_{F}}\right)$ can be obtained from $\mathcal{A}\left(N_{q, q_{F}}\right)$ by normalizing it and connecting the final state to the initial state by an epsilon transition [17. 


\section{Learning $\omega$-Regular Languages}

In this section, we present an algorithm that learns an unknown $\omega$-regular language and generates a nondeterministic Büchi automaton which recognizes $L$ as the result. There are well-known and well-studied algorithms for learning a deterministic finite automaton (DFA) 2218]. We propose an approach which uses the $\mathrm{L}^{*}$ algorithm 2 as the basis for learning an unknown $\omega$-regular language $L$.

The idea behind $\mathrm{L}^{*}$ is learning by experimentation. The learner has the ability to make membership queries. An oracle (a teacher who knows the target language), on any input word $v$, returns a yes-or-no answer depending on whether $v$ belongs to the target language. The learning algorithm thus chooses particular inputs to classify, and consequently makes progress. The learner also has the ability to make equivalence queries. A conjecture language is guessed by the learner, which will then be verified by the teacher through an equivalence check against the target language. The teacher returns yes when the conjecture is correct, or no accompanied by a counterexample to the equivalence of the conjecture and the target language. This counterexample can be a positive counterexample (a word that belongs to the target language but does not belong to the conjecture language) or a negative counterexample (a word that does not belong to the the target language but belongs to the conjecture language). We refer the reader unfamiliar with $\mathrm{L}^{*}$ to 2 for more information on the algorithm.

The goal of our learning algorithm is to come up with a nondeterministic Büchi automaton that recognizes an unknown $\omega$-regular language $L$. We assume that there is a teacher who can correctly answer the membership and equivalence queries on $L$ as discussed above. The idea is to learn the language $L_{\$}$ instead of learning $L$ directly. One can reuse the core of the $L^{*}$ algorithm here, but many changes have to be made. The reason is that the membership and equivalence queries allowed in the setting of our algorithm are for the $\omega$-regular language $L$ and not for the regular language $L_{\$}$. One has to translate the queries and their responses back and forth from the $L_{\$}$ level to the $L$ level.

Membership Queries: The L* algorithm frequently needs to ask questions of the form: "does the string $w$ belong to the target language $L_{\$}$ ?". We need to translate this query into one that can be posed to our teacher. The following simple steps perform this task:

1. Does $w$ belong to $\Sigma^{*} \$ \Sigma^{+}$? If no, then the answer is "NO". If yes, then go to the next step.

2. Let $w=u \$ v$. Does $u v^{\omega}$ belong to $L$ ? if no, then the answer is "NO". If yes, then the answer is "YES".

We know that $L_{\$} \subseteq \Sigma^{*} \$ \Sigma^{+}$which helps us filter out some strings without asking the teacher. If we have $w \in \Sigma^{*} \$ \Sigma^{+}$, then $w$ is of the form $u \$ v$ which corresponds to the ultimately periodic word $u v^{\omega}$. The teacher can respond to the membership query by checking whether $u v^{\omega}$ belongs to $L$. The answer to this query indicates whether $u \$ v$ should belong to our current conjecture. Note that by the definition of $L_{\$}$, we have $u \$ v \in L_{\$} \Leftrightarrow u v^{\omega} \in L$. 
Equivalence Queries: L* generates conjecture DFAs that need to be verified, and therefore a question of the form "Is the conjecture language $M_{i}$ equivalent to the target language $L_{\S}$ ?" needs to be asked. We need to translate this query into an equivalent one that can be posed to the teacher:

1. Is $M_{i}$ a subset of $\Sigma^{*} \$ \Sigma^{+}$? If no, get the counterexample and continue with $L^{*}$. If yes, then go the next step.

2. Is $\omega\left(M_{i}\right)$ (the corresponding $\omega$-regular language of $M_{i}$ ) equivalent to $L$ ? If yes, we are done. If no, we get an ultimately periodic word $c$ that is a (negative or positive) counterexample to the equivalence check. Return "NO" and a finitary interpretation of $c$ (described below) to L*.

Again, the $M_{i} \subseteq \Sigma^{*} \$ \Sigma^{+}$check works as a preliminary test to filter out conjectures that are obviously not correct. If a conjecture language (DFA) $M_{i}$ passes the first test, we construct its corresponding Büchi automaton $\omega\left(M_{i}\right)$. The teacher can then respond by checking the equivalence between $L$ and $\omega\left(M_{i}\right)$. If they are not equivalent, the teacher will return a counterexample to the equivalence of the two languages. In order to proceed with $\mathrm{L}^{*}$, we have to translate these $\omega$-words to finite words that are counterexamples to the equivalence of $M_{i}$ and $L_{\$}$. To do this, for the counterexample $u v^{\omega}$, we construct a DFA that accepts $[u \$ v]_{\varrho}$. There are two cases for each counterexample $u v^{\omega}$ :

- The word $u v^{\omega}$ is a positive counterexample: the word $u v^{\omega}$ should be in $\omega\left(M_{i}\right)$ but is not. Since $u v^{\omega} \notin \omega\left(M_{i}\right)$, by Proposition 4 , $[u \$ v]_{\cong} \nsubseteq M_{i}$ and there exists a word $u^{\prime} \$ v^{\prime} \in[u \$ v]_{\varrho}$ such that $u^{\prime} \$ v^{\prime}$ is not in $M_{i}$. Then $u^{\prime} \$ v^{\prime}$ can serve as an effective positive counterexample for the $\mathrm{L}^{*}$ algorithm. To find $u^{\prime} \$ v^{\prime}$, it suffices to check the emptiness of the language $[u \$ v]_{\varrho}-M_{i}$. There are various ways in which one can compute $[u \$ v]_{\Perp}$. One way is by direct construction of a DFA accepting $[u \$ v]_{\cong}$ from the Büchi automaton that accepts the language containing a single word $u v^{\omega}$. There is a detailed description of this construction in [5] (note that although this construction has an exponential blow up in general, in this special case it is linear). We use a different construction in our implementation which is presented in [9].

- The word $u v^{\omega}$ is a negative counterexample: the word $u v^{\omega}$ should not be in $\omega\left(M_{i}\right)$, but it is. Since $u v^{\omega} \in L$, by Proposition 3, $[u \$ v] \triangleq \cap M_{i} \neq \emptyset$ and there exists a word $u^{\prime} \$ v^{\prime} \in[u \$ v]_{\varrho}$ such that $u^{\prime} \$ v^{\prime} \in M_{i}$. One can find this word by checking emptiness of $M_{i} \cap[u \$ v] \triangleq$. Then $u^{\prime} \$ v^{\prime}$ works as a proper negative counterexample for the $\mathrm{L}^{*}$ algorithm.

Here is why the above procedure works: A conjecture $M$ may not be saturated by $\stackrel{\circ}{=}$. Consider the case presented in Figure 1(a). There are four equivalence classes: $\left[u_{1} \$ v_{1}\right]_{\varrho}$ is contained in $M,\left[u_{2} \$ v_{2}\right]_{\varrho}$ and $\left[u_{3} \$ v_{3}\right]_{\varrho}$. have intersections with $M$ but are not contained in it, and $\left[u_{4} \$ v_{4}\right]_{\cong}$ is completely outside $M$. Now assume $L^{\prime}=\omega(M)$ (as defined by (4)) is the $\omega$-regular language corresponding to $M$. Proposition 4 implies that $u_{1} v_{1}^{\omega} \in L^{\prime}$. Proposition 3 implies that $u_{4} v_{4}^{\omega} \notin L^{\prime}$. However, one cannot state anything about $u_{2} v_{2}^{\omega}$ and $u_{3} v_{3}^{\omega}$ with certainty; they may or may not be in $L^{\prime}$. Let us assume (for the sake of the argument) that 


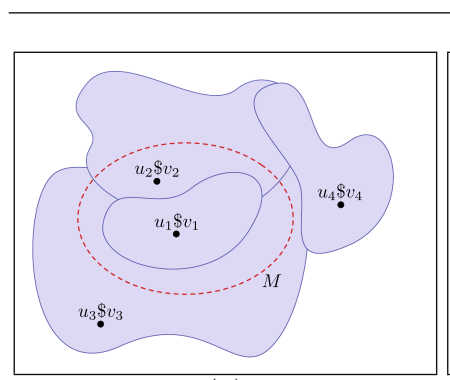

(a)

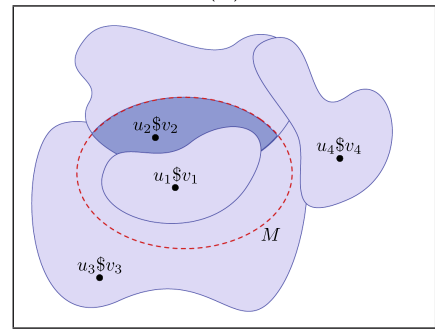

(c)

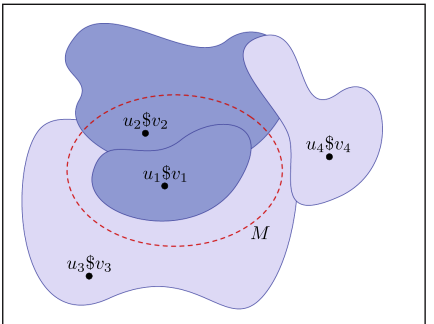

(b)

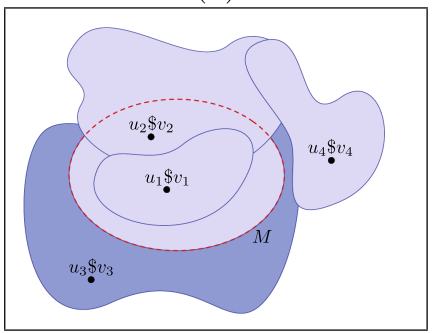

(d)

Fig. 1. The Case of Non-saturation

$u_{2} v_{2}^{\omega} \in L^{\prime}$ and $u_{3} v_{3}^{\omega} \notin L^{\prime}$. This means that $L_{\$}^{\prime}$ (which is not equivalent to $M)$ is actually the shaded area in Figure 11(b). Now, if $L^{\prime}$ is not the correct conjecture $\left(L^{\prime} \neq L\right)$, one will end up with an $\omega$-word $u v^{\omega}$ as a counterexample. As mentioned above, we have one of the following two cases:

(1) The word $u v^{\omega}$ is a negative counterexample $\left(u v^{\omega} \in \omega(M)\right.$ and $\left.u v^{\omega} \notin L\right)$. There are two possibilities for the class $[u \$ v]_{\circ}$ :

- $[u \$ v]_{\triangleq} \subseteq M$ : This case is rather trivial. Any word in $[u \$ v]_{\triangleq}$, including $u \$ v$, belongs to $M$ while it should not. Therefore, $u \$ v$ can serve as a proper negative counterexample for the next iteration of $\mathrm{L}^{*}$.

- $[u \$ v]_{\triangleq} \nsubseteq M$ : This case is more tricky. This means that $u_{2} v_{2}^{\omega}$ was wrongly included in $\omega(M)$. But since some of the strings in $[u \$ v]_{\triangleq}$ do not belong to $M$, an arbitrary string from $[u \$ v]$ 。 does not necessarily work as a negative counterexample for the next iteration of $\mathrm{L}^{*}$. One has to find a string which is in both $[u \$ v]_{\triangleq}$ and $M$, which means it belongs to $[u \$ v]_{\triangleq} \cap M$. The shaded area in Figure 1(c) demonstrates this set for the example. Note that $[u \$ v]_{\triangleq} \cap M$ cannot be empty; by Proposition 3 , $[u \$ v]_{\cong} \cap M=\emptyset$ implies that $u v^{\omega} \notin L$ which is a contradiction.

(2) The word $u v^{\omega}$ is a positive counterexample $\left(u v^{\omega} \notin \omega(M)\right.$ and $\left.u v^{\omega} \in L\right)$. There are two possibilities for the class $[u \$ v]_{\text {。 }}$ :

- $[u \$ v]_{\triangleq} \cap M=\emptyset$ : This case is rather trivial. All words in $[u \$ v]_{\triangleq}$, including $u \$ v$, do not belong to $M$ while they should. Therefore, $u \$ v$ can serve as a proper positive counterexample for the next iteration of $\mathrm{L}^{*}$. 
- $[u \$ v]_{\triangleq} \cap M \neq \emptyset$ : This case is more tricky. This means that $u_{3} v_{3}^{\omega}$ was wrongly left out of $\omega(M)$. But since some of the strings in $[u \$ v]_{\text {。 do }}$ belong to $M$, an arbitrary string from that class is not necessarily going to work as a proper positive counterexample for the next iteration of $\mathrm{L}^{*}$. We have to make sure to find one that is in $[u \$]_{\cong}$ but not in $M$. The

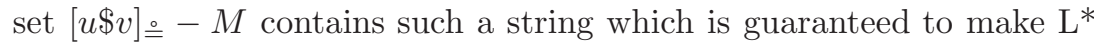
progress. The shaded area in Figure 1(d) demonstrates this set for the

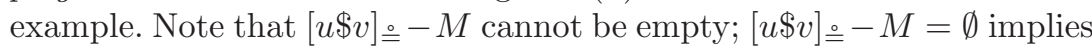
that $[u \$ v]_{\triangleq} \subseteq M$ in which case by Proposition 4, we have $u v^{\omega} \in L$, which is a contradiction.

Below, we give a more technical description of our algorithm followed by an example for greater clarity.

Definition 3. An observation table is a tuple $\langle S, E, T\rangle$ where $S$ is a set of prefixclosed words in $\Sigma^{*}$ such that each word in $S$ represents a syntactic right congruence class of $L_{\$}, E$ is a set of suffix-closed strings in $\Sigma^{*}$ such that each word in $E$ is a distinguishing word, and $T:(S \cup S \Sigma) \times E \longrightarrow\{-,+\}$ is defined as

$$
T(\alpha, \sigma)=\left\{\begin{array}{l}
+ \text { if } \alpha \sigma \in L_{\$} \\
- \text { if } \alpha \sigma \notin L_{\$} .
\end{array}\right.
$$

An observation table is closed if for every word $s^{\prime} \in S \Sigma$, there exists a word $s \in S$ such that $T(s, \bullet)=T\left(s^{\prime}, \bullet\right)$ (where $T(s, \bullet)$ indicates the row of the table which starts with $s$ ).

The goal of $\mathrm{L}^{*}$ here is to learn $L_{\$}$ for an unknown $\omega$-language $L$ on alphabet $\Sigma \cup\{\$\}$. Our initial setting is the same as $\mathrm{L}^{*}$; the distinguishing experiment set $E=\{\lambda\}$ and the congruence class set $S=\{\lambda\}$. We fill the table by asking membership queries for each pair of strings $(\alpha, \sigma) \in(S \cup S \Sigma) \times E$; a "NO" response sets $T(\alpha, \sigma)=-$, and a "YES" response sets $T(\alpha, \sigma)=+$. Note that the membership queries are translated as discussed above to a format appropriate for the teacher.

When the observation table is closed, a conjecture DFA $A=\left(S, \Sigma, q_{0}, F, \delta\right)$, where $Q=\{u \mid u \in S\}, q_{0}=\lambda, \delta=\left\{\left(u, a, u^{\prime}\right) \mid u^{\prime} \in S \wedge T\left(u^{\prime}, \bullet\right)=T(u a, \bullet)\right\}$, and $F=\{u \mid T(u, \lambda)=+\}$ is constructed from the table.

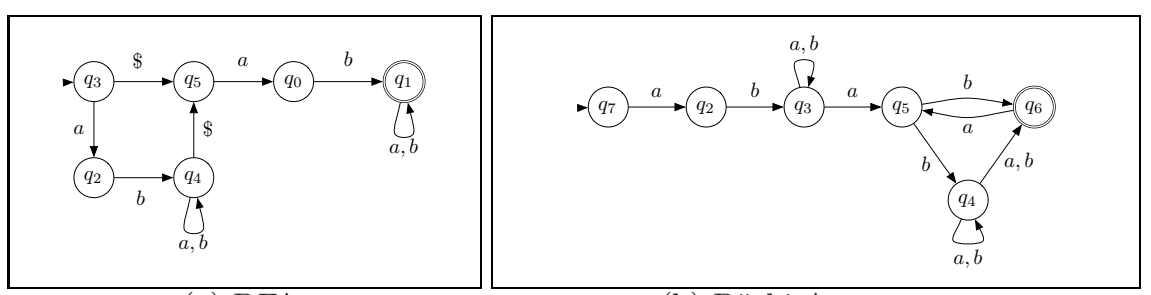

(a) DFA.

(b) Büchi Automaton.

Fig. 2. First Iteration 
We then check if $M_{0}=L(A)$ is a subset of $\Sigma^{*} \$ \Sigma^{+}$. If not, there is a counterexample in $L\left(A_{\$}\right) \cap \overline{\Sigma^{*} \$ \Sigma^{+}}$from the language containment check, all of whose suffixes are added to the set of distinguishing words $E$. If $M_{0} \subseteq \Sigma^{*} \$ \Sigma^{+}$, we construct a Büchi automaton $B$ based on $A$ (see Section 3 ), and perform the equivalence check. The counterexamples are interpreted (as discussed above) and the appropriate counterexamples are added to set $E$. We then proceed to another iteration of this algorithm, until the target language is found.

Example 1. We demonstrate our algorithm by showing the steps performed on a simple example. Assume that the target language is $a b\left((a+b)^{*} a\right)^{\omega}$. This $\omega$ expression corresponds to the liveness property: " $a$ happens infinitely often in a computation with the prefix $a b$ " which cannot be learned using any of the existing algorithms.

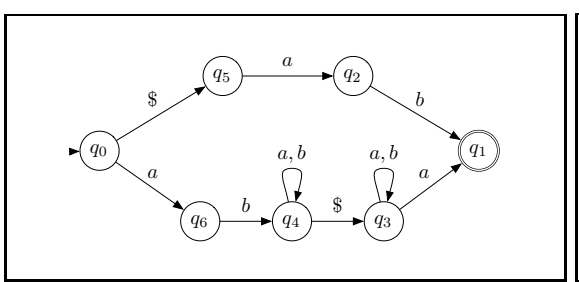

(a) DFA.

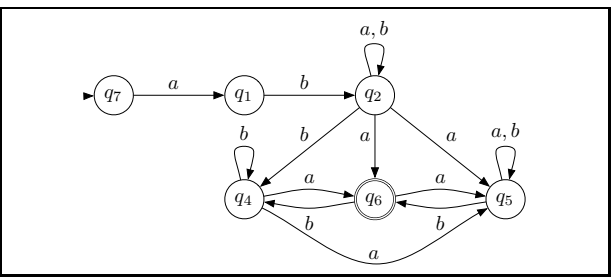

(b) Büchi Automaton.

Fig. 3. Second Iteration

Table 1(a) shows the closed observation from the first iteration of our algorithm. Figure 2(a) demonstrates the DFA that corresponds to this observation table, and Figure 2(b) demonstrates the Büchi automaton constructed form this DFA. The first conjecture is not correct; the word $a b(a)^{\omega}$ belongs to the target language, but is not accepted by the automaton in Figure 2(b). Therefore, the algorithm goes into a second iteration. The counterexample is translated into one appropriate for the $\mathrm{L}^{*}(a b \$ a)$, and all its suffixes are added to the top row of the table. Table 1(b) is the closed table which we acquire after adding the counterexample. Figure 3(a) shows the DFA corresponding to this table, and Figure 3(b) shows the Büchi automaton constructed based on this DFA. This Büchi automaton passes the equivalence check and the algorithm is finished learning the target language.

Complexity: Note that for the target $\omega$-regular language $L$, our learning algorithm terminates by learning $L_{\$}$ (and hence $L$ ). We can show that our learning algorithm is polynomial (in terms of number of queries performed) on the size of $L_{\$}$ (and the size of the counter examples). However, one can show that $L_{\$}$, in the worst case, can be exponentially bigger that the minimal Büchi automaton accepting $L$. We refer the interested reader to 9 for a more detailed discussion. 


\section{Optimizations}

In this section, we briefly discuss some practical optimizations that we have added to our implementation of the algorithm presented in Section 4 to gain a more efficient learning tool.

Equivalence query as the last resort: The equivalence query for an $\omega$-regular language is expensive, even more than equivalence checking for regular languages. The main reason is that it requires complementing the Büchi automaton, which has a proven lower bound of $2^{O(n \log n)}$ [16]. Therefore, having fewer equivalence queries speeds up the process of learning. For each conjecture DFA $A$ that is built during an iteration of the algorithm (more specifically, the incorrect conjectures),

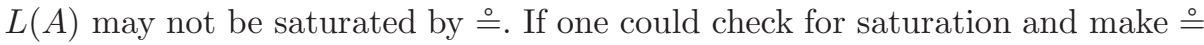
saturate $L(A)$ by adding/removing words, one could avoid going through with an (expensive) equivalence check that will most probably have a "NO" response. Unfortunately, there is no known way of effectively checking for saturation. But all is not lost. One can construct another DFA $A^{\prime}$ where $L\left(A^{\prime}\right)=\left(\Sigma^{*} \$ \Sigma^{+}\right) \cap$ $\overline{L(A)}$. Since $A$ is deterministic, $A^{\prime}$ can easily be constructed. Let $B$ and $B^{\prime}$ be respectively the corresponding Büchi automata for $A$ and $A^{\prime}$. If $L(B) \cap L\left(B^{\prime}\right) \neq \emptyset$ then there is $u v^{\omega} \in L(B) \cap L\left(B^{\prime}\right)$, and we know that only a part of the equivalence class $[u \$ v]_{\circ}$ is in $L(A)$ and the rest of it is in $L\left(A^{\prime}\right)$. To decide whether the class should go into $L(A)$ (the conjecture) completely, or be altogether removed from

Table 1. Observation Tables

\begin{tabular}{|l||c|c|c|c|c|c|c|}
\hline & $\lambda$ & $a b$ & $b$ & $\$ a b$ & $a \$ a b$ & $a b a \$ a b$ & $b a \$ a b$ \\
\hline$\lambda$ & - & - & - & + & - & - & + \\
$a$ & - & - & - & - & - & + & - \\
$b$ & - & - & - & - & - & - & - \\
$\$$ & - & - & + & - & - & - & - \\
$a b$ & - & - & - & + & + & + & + \\
$\$ a$ & - & + & - & - & - & - & - \\
$\$ a b$ & + & + & + & - & - & - & - \\
\hline$a a$ & - & - & - & - & - & - & - \\
$a \$$ & - & - & - & - & - & - & - \\
$b a$ & - & - & - & - & - & - & - \\
$b b$ & - & - & - & - & - & - & - \\
$b \$$ & - & - & - & - & - & - & - \\
$\$ b$ & - & - & - & - & - & - & - \\
$\$ \$$ & - & - & - & - & - & - & - \\
$a b a$ & - & - & - & + & + & + & + \\
$a b b$ & - & - & - & + & + & + & + \\
$a b \$$ & - & - & + & - & - & - & - \\
$\$ a a$ & - & - & - & - & - & - & - \\
$\$ a \$$ & - & - & - & - & - & - & - \\
$\$ a b a$ & + & + & + & - & - & - & - \\
$\$ a b b$ & + & + & + & - & - & - & - \\
$\$ a b \$$ & - & - & - & - & - & - & - \\
\hline
\end{tabular}

(a) First Iteration.

\begin{tabular}{|l||c|c|c|c|c|c|c|c|c|c|c|}
\hline & $\lambda$ & $a b$ & $b$ & $\$ a b$ & $a \$ a b$ & $a b a \$ a b$ & $b a \$ a b$ & $a$ & $\$ a$ & $b \$ a$ & $a b \$ a$ \\
\hline$\lambda$ & - & - & - & + & - & - & + & - & - & - & + \\
$a$ & - & - & - & - & - & + & - & - & - & + & - \\
$b$ & - & - & - & - & - & - & - & - & - & - & - \\
$\$$ & - & - & + & - & - & - & - & - & - & - & - \\
$a b$ & - & - & - & + & + & + & + & - & + & + & + \\
$\$ a$ & - & + & - & - & - & - & - & - & - & - & - \\
$\$ a b$ & + & + & + & - & - & - & - & + & - & - & - \\
$a b \$$ & - & - & + & - & - & - & - & + & - & - & - \\
\hline$a a$ & - & - & - & - & - & - & - & - & - & - & - \\
$a \$$ & - & - & - & - & - & - & - & - & - & - & - \\
$b a$ & - & - & - & - & - & - & - & - & - & - & - \\
$b b$ & - & - & - & - & - & - & - & - & - & - & - \\
$b \$$ & - & - & - & - & - & - & - & - & - & - & - \\
$\$ b$ & - & - & - & - & - & - & - & - & - & - & - \\
$\$ \$$ & - & - & - & - & - & - & - & - & - & - & - \\
$a b a$ & - & - & - & + & + & + & + & - & + & + & + \\
$a b b$ & - & - & - & + & + & + & + & - & + & + & + \\
$\$ a a$ & - & - & - & - & - & - & - & - & - & - & - \\
$\$ a \$$ & - & - & - & - & - & - & - & - & - & - & - \\
$\$ a b a$ & + & + & + & - & - & - & - & + & - & - & - \\
$\$ a b b$ & + & + & + & - & - & - & - & + & - & - & - \\
$\$ a b \$$ & - & - & - & - & - & - & - & - & - & - & - \\
$a b \$ a$ & + & + & + & - & - & - & - & + & - & - & - \\
$a b \$ b$ & - & - & + & - & - & - & - & + & - & - & - \\
$a b \$ \$$ & - & - & - & - & - & - & - & - & - & - & - \\
\hline
\end{tabular}

(b) Second Iteration. 
it, we can pose a membership query for $u v^{\omega}$ to the teacher. If $u v^{\omega} \in L$, then the class should belong to the conjecture, and therefore any word in $[u \$ v] \triangleq \cap L\left(A^{\prime}\right)$ works as a positive counterexample for the next iteration of $\mathrm{L}^{*}$. If $u v^{\omega} \notin L$ then any word in $[u \$ v]_{\varrho} \cap L(A)$ can serve as a negative counterexample for the next iteration of the $\mathrm{L}^{*}$. This check is polynomial in the size of $A$, and saves us an unnecessary equivalence query.

Minimization and Simplification: Our algorithm constructs and handles many DFAs during the construction of the Büchi automaton from the conjecture DFA (from $M_{q}$ 's and $N_{q, q_{f}}$ 's). Hence, the algorithm can benefit from minimizing all those DFAs in order to reduce the overhead of working with them later on. DFA minimization can be done very efficiently; the complexity is $n \log n[12$, where $n$ is the size of the source DFA.

When the conjecture Büchi automaton is built, another useful technique is to simplify the Büchi automaton by detecting a simulation relation between states 19. Intuitively, a state $p$ simulates another state $q$ in the Büchi automaton if all accepting traces starting from $q$ are also accepting traces starting from $p$. If $p$ simulates $q$ and both transitions $r \stackrel{a}{\longrightarrow} p$ and $r \stackrel{a}{\longrightarrow} q$ are in the automaton, then $r \stackrel{a}{\longrightarrow} q$ can be safely removed without changing the language of the Büchi automaton. Furthermore, if $p$ and $q$ simulate each other, then after redirecting all of $q$ 's incoming transitions to $p, q$ can be safely removed. This technique is useful for reducing the size of the result automaton, because the structures of $M_{q}$ and $N_{q, q_{f}}^{\omega}$ are usually very similar, which provides good opportunities for finding simulation relations.

\section{Preliminary Experimental Results}

We have implemented our algorithm using JAVA. DFA operations are delegated to the dk.brics.automaton package, and the Büchi automaton equivalence checking function is provided by the GOAL tool [20].

We check the performance of our tool by learning randomly generated $\omega$ regular languages. More specifically, we combine the following 5 steps to get a target Büchi automaton:

1. Randomly generate LTL formulas with a length of 6 and with 1 or 2 propositions (which produces respectively Büchi automata with $|\Sigma|=2$ and 4).

2. If the formula appeared before, discard it and go back to step 1.

Table 2. Results for Randomly Generated Temporal Formulas

\begin{tabular}{|l|c|c|c||c|c|c|}
\hline & \multicolumn{3}{|c||}{$|\Sigma|=2$} & \multicolumn{3}{c|}{$|\Sigma|=4$} \\
\cline { 2 - 7 } & Avg & Min & Max & Avg & Min & Max \\
\hline Target BA recognizing L & 5.3 & 5 & 7 & 5.34 & 5 & 10 \\
Learned DFA & 7.98 & 3 & 21 & 8.7 & 5 & 16 \\
Learned BA & 6.78 & 3 & 11 & 12.92 & 5 & 35 \\
Learned BA (after simplification) & 5.36 & 2 & 8 & 9.38 & 3 & 24 \\
\hline
\end{tabular}

(Unit: number of states) 
3. Use the LTL2BA [10] algorithm to make them Büchi automata.

4. Apply the simplification [19] to make the Büchi automata as small as possible.

5. If the size of the automaton is smaller than 5 , discard it and go to step 1 .

Note that the combination of these steps does not guarantee the minimality of the resulting Büchi automaton.

Table 2 presents the performance of our algorithm on these randomly generated $\omega$-regular languages. The sizes of the learned automata are compared with the sizes of the target automata. The result shows that the size the learned automaton is comparable with the size of the target automaton. Table 2 presents a summary of the results of 100 learning tasks. We have 50 are with $|\Sigma|=2$ and another half of them with $|\Sigma|=4$.

Table 3. Effectiveness for learning automata from selected temporal formulas

\begin{tabular}{|c|c|c|c|c|c|c|c|c|c|c|c|}
\hline \multirow{2}{*}{$\begin{array}{l}\text { Property } \\
\text { Classes }\end{array}$} & \multirow{2}{*}{$\begin{array}{l}\text { Canonical } \\
\text { Formulas }\end{array}$} & \multicolumn{2}{|c|}{\begin{tabular}{|l|} 
Target \\
\end{tabular}} & \multicolumn{2}{|c|}{ Learned } & \multirow{2}{*}{$\begin{array}{c}\text { Responsive } \\
\text { Formulas }\end{array}$} & \multicolumn{2}{|c|}{\begin{tabular}{|l|} 
Target \\
\end{tabular}} & \multicolumn{2}{|c|}{ Learned } & \multirow{2}{*}{$\begin{array}{l}\subseteq \mathrm{DBB} \cap \\
\text { coDB? }\end{array}$} \\
\hline & & $\overline{\mathrm{St}}$ & Trans & St & Trans & & $\overline{\mathrm{St}}$ & Trans & $\overrightarrow{\mathrm{St}}$ & Trans & \\
\hline Reactive & $\mathbf{F G} p \vee \mathbf{G F} q$ & 5 & 26 & 7 & \begin{tabular}{|l|}
37 \\
\end{tabular} & $\mathbf{G F} p \rightarrow \mathbf{G F} q$ & 5 & 26 & 7 & 37 & No \\
\hline Persistence & $\mathbf{F G} p$ & 2 & 4 & 3 & 7 & $\mathbf{G}(p \rightarrow \mathbf{F} \mathbf{G} q)$ & 4 & 18 & 11 & 43 & No \\
\hline Recurrence & GF $p$ & 2 & 12 & 3 & 9 & $\mathbf{G}(p \rightarrow \mathbf{F} q)$ & 3 & 12 & 13 & 65 & No \\
\hline Obligation & $\mathbf{G} p \vee \mathbf{F} q$ & 4 & 15 & 6 & 25 & $\mathbf{F} p \rightarrow \mathbf{F} q$ & 4 & 15 & 6 & 25 & Yes \\
\hline Safety & $\mathbf{G} p$ & 2 & 2 & 2 & 3 & $p \rightarrow \mathbf{G} q$ & 3 & 9 & 9 & 32 & Yes \\
\hline Guarantee & $\mathbf{F} p$ & 2 & 4 & 3 & 8 & $p \rightarrow \mathbf{F} q$ & 3 & 12 & 4 & 20 & Yes \\
\hline
\end{tabular}

On a different note, we present the performance of our algorithm on learning properties that are often used in verification. Table 3 presents the result of these experiments. The target languages are described by temporal formulas selected from Manna and Pnueli [15] and classified according to the hierarchy of temporal properties which they proposed in the same paper. We translate those temporal formulas to Büchi automata by the LTL2BA algorithm. The first column of the table lists the six classes of the hierarchy. We select two temporal formulas for each class 2 . One of them is a formula in "canonical form" 3 and the other is a formula in "responsive form" 4 . Maler and Pnueli's algorithm 14 can only handle the bottom three levels of that hierarchy. Their algorithm cannot handle some important properties such as progress $\mathbf{G}(p \rightarrow \mathbf{F} q)$ and strong fairness $\mathbf{G F} p \rightarrow \mathbf{G F} q$, which can be handled by our algorithm.

\section{Conclusions and Future Work}

We have extended the learning paradigm of the infinitary languages by presenting an algorithm to learn an arbitrary $\omega$-regular language $L$ over an alphabet $\Sigma$.

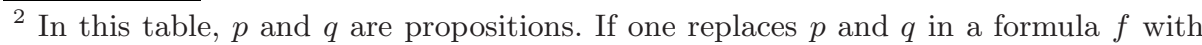
temporal formulas containing only past operators, $f$ still belongs to the same class.

3 The canonical formula is a simple representative formula for each class.

${ }^{4}$ A responsive formula usually contains two propositions $p$ and $q$. The proposition $p$ represents a stimulus and $q$ is a response to $p$.
} 
Our main result is an algorithm to learn a nondeterministic Büchi automaton that recognizes an unknown $\omega$-regular language by learning a unique projection of it $\left(L_{\$}\right)$ on $\Sigma^{*}$ using the $\mathrm{L}^{*}[2]$ algorithm. We also presented preliminary experimental results that suggest that algorithms performs well on small examples.

In the future, we would like to extend our experiments by learning bigger Büchi automata. We would also like to use this learning algorithm as a core of a compositional verification tool to equip the tool with the capability to check liveness properties that have been missing from such tools so far. One way of improving our algorithm is to find an effective way of checking for saturation, which appears to be difficult and remains unsolved.

\section{References}

1. Alur, R., Madhusudan, P., Nam, W.: Symbolic compositional verification by learning assumptions. In: Etessami, K., Rajamani, S.K. (eds.) CAV 2005. LNCS, vol. 3576, pp. 548-562. Springer, Heidelberg (2005)

2. Angluin, D.: Learning regular sets from queries and counterexamples. Information and Computation 75(2), 87-106 (1987)

3. Arnold, A.: A syntactic congruence for rational omega-language. Theoretical Computer Science 39, 333-335 (1985)

4. Büchi, J.R.: On a decision method in restricted second-order arithmetic. In: Proceedings of the 1960 International Congress on Logic, Methodology and Philosophy of Science, pp. 1-11 (1962)

5. Calbrix, H., Nivat, M., Podelski, A.: Ultimately periodic words of rational $\omega$ languages. In: Main, M.G., Melton, A.C., Mislove, M.W., Schmidt, D., Brookes, S.D. (eds.) MFPS 1993. LNCS, vol. 802, pp. 554-566. Springer, Heidelberg (1994)

6. Calbrix, H., Nivat, M., Podelski, A.: Sur les mots ultimement périodiques des langages rationnels de mots infinis. Comptes Rendus de l'Académie des Sciences 318, 493-497 (1994)

7. Chaki, S., Clarke, E., Sinha, N., Thati, P.: Automated assume-guarantee reasoning for simulation conformance. In: Etessami, K., Rajamani, S.K. (eds.) CAV 2005. LNCS, vol. 3576, pp. 534-547. Springer, Heidelberg (2005)

8. Cobleigh, J.M., Giannakopoulou, D., Păsăreanu, C.S.: Learning assumptions for compositional verification. In: Garavel, H., Hatcliff, J. (eds.) TACAS 2003. LNCS, vol. 2619, pp. 331-346. Springer, Heidelberg (2003)

9. Farzan, A., Chen, Y., Clarke, E., Tsay, Y., Wang, B.: Extending automated compositional verification to the full class of omega-regular languages. Technical Report CMU-CS-2008-100, Carnegie Mellon University, Department of Computer Science (2008)

10. Gastin, P., Oddoux, D.: Fast LTL to Büchi automata translations. In: Berry, G., Comon, H., Finkel, A. (eds.) CAV 2001. LNCS, vol. 2102, pp. 53-65. Springer, Heidelberg (2001)

11. Gupta, A., McMillan, K.L., Fu, Z.: Automated assumption generation for compositional verification. In: Damm, W., Hermanns, H. (eds.) CAV 2007. LNCS, vol. 4590, pp. 420-432. Springer, Heidelberg (2007)

12. Hopcroft, J.E.: A n logn algorithm for minimizing states in a finite automaton. Technical report, Stanford University (1971)

13. Hopcroft, J.E., Ullman, J.D.: Introduction to Automata Theory, Languages and Computation. Addison-Wesley, Reading (1979) 
14. Maler, O., Pnueli, A.: On the learnability of infinitary regular sets. Information and Computation 118(2), 316-326 (1995)

15. Manna, Z., Pnueli, A.: A hierarchy of temporal properties. Technical Report STANCS-87-1186, Stanford University, Department of Computer Science (1987)

16. Michel, M.: Complementation is more difficult with automata on infinite words. In: CNET, Paris (1988)

17. Perrin, D., Pin, J.E.: Infinite Words: Automata, Semigroups, Logic and Games. Academic Press, London (2003)

18. Rivest, R.L., Schapire, R.E.: Inference of finite automata using homing sequences. Information and Computation 103(2), 299-347 (1993)

19. Somenzi, F., Bloem, R.: Efficient Büchi automata from LTL formulae. In: Emerson, E.A., Sistla, A.P. (eds.) CAV 2000. LNCS, vol. 1855, pp. 248-263. Springer, Heidelberg (2000)

20. Tsay, Y., Chen, Y., Tsai, M., Wu, K., Chan, W.: GOAL: A Graphical Tool for Manipulating Büchi Automata and Temporal Formulae. In: Grumberg, O., Huth, M. (eds.) TACAS 2007. LNCS, vol. 4424, pp. 466-471. Springer, Heidelberg (2007)

21. Van, D.L., Le Saëc, B., Litovsky, I.: Characterizations of rational omega-languages by means of right congruences. Theor. Comput. Sci. 143(1), 1-21 (1995) 